\title{
Enclosure size and the use of local and global geometric cues for reorientation
}

\author{
Bradley R. Sturz • Martha R. Forloines $\cdot$ Kent D. Bodily
}

Published online: 5 January 2012

(C) Psychonomic Society, Inc. 2011

\begin{abstract}
Multiple spatial cues are utilized to orient with respect to the environment, but it remains unclear why feature (i.e., objects in the environment) and geometric (i.e., shape of the environment) cues are differentially influenced by enclosure size, and the extent to which local (i.e., wall lengths and corner angles) and global (i.e., principal axis of space) geometric cues are influenced by enclosure size. In the present study, we investigated the extent to which environmental size influenced the use of corner angle (i.e., a local geometric cue) and the principal axis of space (i.e., a global geometric cue) for reorientation. We developed an orientation task that allowed the manipulation of enclosure size during training and the isolation of the use of the principal axis of space during testing. Participants were trained to respond to a location in either a small or a large trapezoid-shaped enclosure uniquely specified by both local (i.e., wall lengths and corner angles) and global (i.e., principal axis of space) geometric cues. During testing, we presented both groups with a small and large rectangle (to assess the use of principal axis of space) and a small and large parallelogram (to asses relative use of corner angles and the principal axis of space when in conflict). Enclosure size influenced the relative use of corner angles but not of the principal axis of space. Results suggest that corner angles function like features and that changes in the use of feature cues are the source of the relative reliance on feature and geometric cues during changes of enclosure size.
\end{abstract}

\footnotetext{
B. R. Sturz • M. R. Forloines $\cdot$ K. D. Bodily

Georgia Southern University,

Statesboro, GA, USA

B. R. Sturz $(\bowtie)$

Department of Psychology, Georgia Southern University,

P.O. Box 8041, Statesboro, GA 30460, USA

e-mail: bradleysturz@georgiasouthern.edu
}

Keywords Virtual Environment · Orientation · Global Geometry · Local Geometry Enclosure Size

Determining current position in space is essential to a process of navigating between locations. In order to investigate this essential component of the navigational process, researchers often utilize an orientation task that involves training participants to locate a particular corner marked with a unique feature in a rectangular enclosure (for a review, see Cheng \& Newcombe, 2005). Following training, aspects of the enclosure are modified to determine the stimuli by which participants orient with respect to the environment (see Cheng, 2008). Pervasive use of this task has revealed that in the absence of the unique features present during training, participants allocate equivalent responses to the trained (i.e., correct) and rotationally equivalent locations at above chance levels (for a review, see Cheng \& Newcombe, 2005). Above chance responding to these "geometrically correct" locations has been taken as evidence that participants learn about the geometric properties (i.e., shape) of the enclosure during training (see Cheng, 2005).

In recent years, other aspects of the rectangular enclosure have been modified between training and testing to delineate the spatial cues by which participants orient with respect to the environment. Such modifications to the enclosure include both size and shape (Kelly, Chiandetti, \& Vallortigara, 2011; Sturz, Gurley, \& Bodily, 2011; Sturz \& Kelly, 2009). Interestingly, participants continue to respond to these geometrically correct locations at above chance levels following changes in shape (Kelly et al., 2011; Sturz et al., 2011) and size (Sturz \& Kelly, 2009). Perhaps more interestingly, changes in enclosure size from training to testing have been shown to influence the relative contribution of feature and geometric cues in the reorientation process (Ratliff \& Newcombe, 2008; Sovrano, 
Bisazza, \& Vallortigara, 2005; Sovrano, Bisazza, \& Vallortigara, 2007; Vallortigara, Feruglio, \& Sovrano, 2005). In short, feature cues appear to exert a greater influence in larger enclosures, whereas geometric cues appear to exert a greater influence in smaller enclosures (see also Miller, 2009).

To date, it remains unclear whether enclosure size is directly influencing the use of feature cues, the use of geometric cues, or both feature and geometric cues because of the difficulty in isolating the use of only feature or geometric cues during reorientation (see Miller, 2009; Sovrano \& Vallortigara, 2006). Specifically, existing research is unable to determine which spatial cue(s) is responsible for the relative use of feature and geometric cues during changes in enclosure size because increases in the use of feature cues result in relative decreases in the use of geometric cues. Reciprocally, increases in the use of geometric cues result in relative decreases in the use of feature cues (Ratliff \& Newcombe, 2008; Sovrano et al., 2005, 2007; Vallortigara et al., 2005).

Despite this unknown, it is clear that both feature and geometric cues are utilized in the reorientation process (for a review, see Cheng \& Newcombe, 2005), and it has been recently suggested that both local (e.g., wall lengths and corner angles) and global (e.g., principal axis of space) geometric cues are also utilized in the reorientation process (Bodily, Eastman,
\& Sturz, 2011; Kelly et al., 2011a; see also Sturz \& Bodily, 2011a; Kelly, Durocher, Chiandetti, \& Vallortigara, 2011). Given the recent evidence that participants are able to utilize both local and global geometric cues, we questioned whether the use of local and global geometric cues was influenced by enclosure size in similar ways as those of feature and geometric cues and, by extension, the source of the relative contribution of local and global geometric cues during changes of enclosure size.

With these questions in mind, the purpose of the present experiment was to determine the influence of enclosure size on the use of corner angle (i.e., a local geometric cue) and the principal axis of space (i.e., a global geometric cue) for reorientation. The use of a virtual environment lent itself well to such an investigation because of the ease in environmental manipulation coupled with the similarity of mechanisms utilized in real and virtual environments (see Sturz, Bodily, Katz, Kelly, 2009; Sturz, Brown, \& Kelly, 2009; Sturz, Kelly, $\&$ Brown, 2010). To this end, we trained two groups of participants to find a location uniquely specified by both local (i.e., short wall left, short wall right, obtuse corner angle) and global (i.e., right side of principal axis) geometric cues in either a small or a large trapezoid-shaped virtual enclosure (see Fig. 1). Following training, we presented participants in both groups with a small and a large rectangle and a small and
Fig. 1 Schematics of training and testing trials for each group. For illustrative purposes, the gray circles mark the position at which participants entered the virtual enclosures for all training and testing trials, unfilled circles indicate invisible response locations, dotted lines represent the principal axis of space

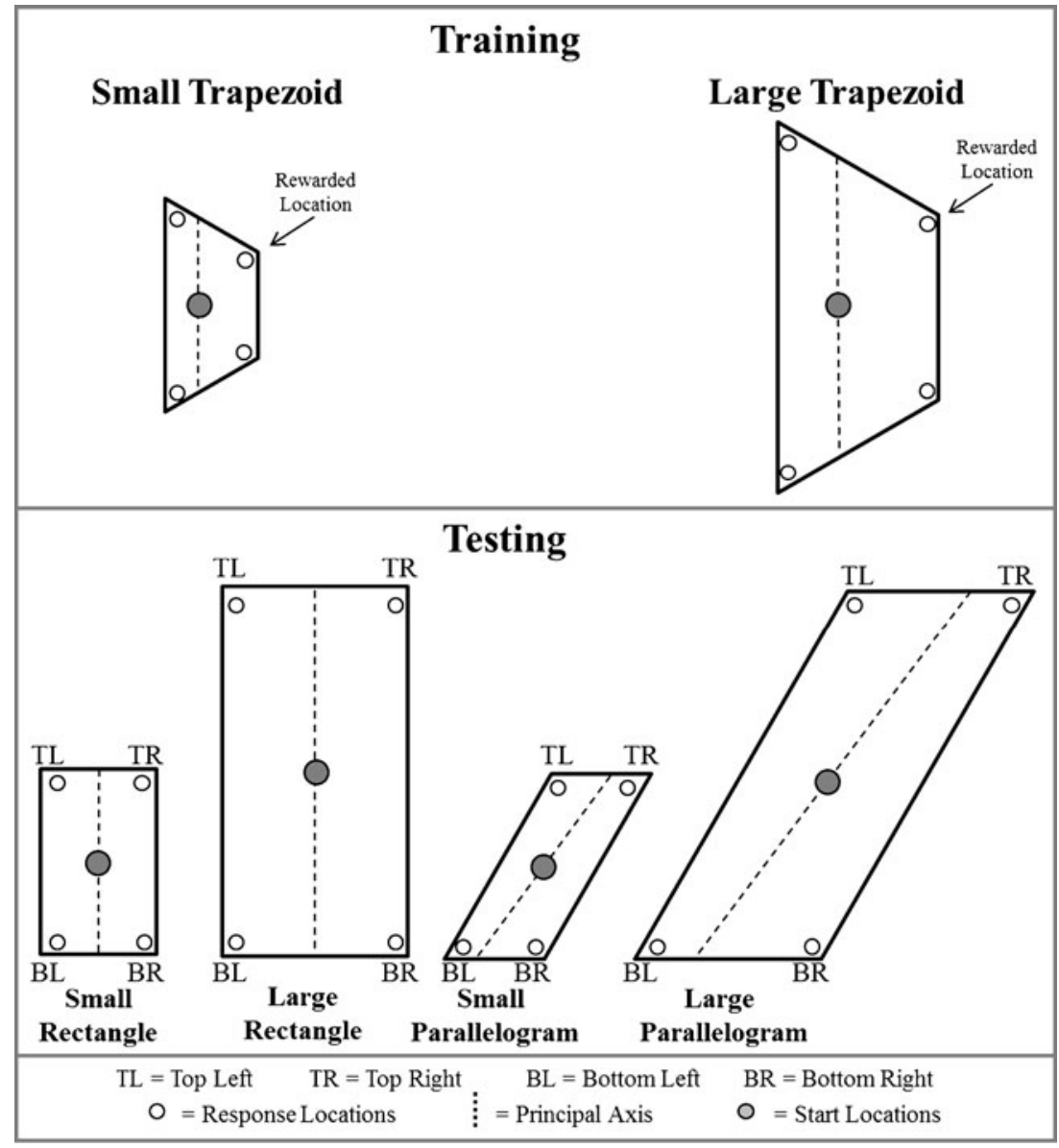


large parallelogram (see Fig. 1). These testing enclosures allowed us to assess for orientation in the absence of useful wall lengths and corner angles (in the rectangles) in order to isolate the use of the principal axis of space. In addition, the testing enclosures allowed us to assess for orientation in the presence of a conflict between trained corner angles and the trained side of the principal axis of space (in the parallelograms).

If corner angles and the principal axis of space are influenced by enclosure size in similar ways as that of feature (i.e., beacons, landmarks) and geometric cues, then participants trained in the small enclosure should allocate more responses to locations specified by the trained side of the principal axis of space than should participants trained in the large enclosure during test trials in the rectangular enclosures. In addition, participants trained in the large enclosure should allocate more responses to locations specified by trained corner angles than should participants trained in the small enclosure during test trials in the parallelogram enclosures. By isolating the use of the principal axis of space for reorientation, should corner angles and the principal axis of space be influenced by enclosure size, our design allows us to determine the source of the relative contribution of local and global geometric cues during changes of enclosure size.

\section{Method}

\section{Participants}

Thirty-six undergraduate students (18 males and 18 females) served as participants. Participants received extra class credit or participated as part of a course requirement.

\section{Apparatus}

An interactive, dynamic three-dimensional virtual environment was constructed and rendered using Valve Hammer Editor and was run on the Half-Life Team Fortress Classic platform. A personal computer, 21-in. flat-screen liquid crystal display (LCD) monitor, gamepad joystick, and speakers served as the interface with the virtual environment. The monitor $(1,152 \times 864$ pixels $)$ provided a first-person perspective of the virtual environment. Participants utilized the joystick on the gamepad to navigate within the environment. Speakers emitted auditory feedback. Experimental events were controlled and recorded using a Half-Life Dedicated Server on an identical personal computer.

Stimuli

Dimensions are long wall(s) $\times$ short walls $\times$ height and are measured in virtual units (vu). Six virtual enclosures were created (see Fig. 1): small trapezoid $(552 \times 276 \times 260 \mathrm{vu}=$ $\sim 14 \times 7 \times 6.6 \mathrm{~m})$, large trapezoid $(1,104 \times 552 \times 260 \mathrm{vu}=$ $\sim 28 \times 14 \times 6.6 \mathrm{~m})$, small rectangle $(552 \times 276 \times 260 \mathrm{vu}=$ $\sim 14 \times 7 \times 6.6 \mathrm{~m})$, large rectangle $(1,104 \times 552 \times 260 \mathrm{vu}=$ $\sim 28 \times 14 \times 6.6 \mathrm{~m})$, small parallelogram $(552 \times 276 \times 260 \mathrm{vu}=$ $\sim 14 \times 7 \times 6.6 \mathrm{~m})$, and large parallelogram $(1,104 \times 552 \times 260$ $\mathrm{vu}=\sim 28 \times 14 \times 6.6 \mathrm{~m})$. All corner angles were $90^{\circ}$ in the rectangle. Corner angles for the trapezoid-shaped enclosures were $60^{\circ}$ for both acute angles and $120^{\circ}$ for both obtuse angles. Corner angles in the parallelograms were also $60^{\circ}$ for both acute angles and $120^{\circ}$ for both obtuse angles. All surfaces were white in color with the exceptions of the floors (gray tile) and the ceilings (black). We delineated four response locations within each enclosure $(48 \times 48 \times 48 \mathrm{vu}=\sim 1.2 \times 1.2 \times 1.2 \mathrm{~m}$, see Fig. 1), but response locations were not visible to participants.

\section{Procedure}

Participants were instructed to navigate to the location that transported them to the next virtual room and to move via the joystick on the gamepad: $\uparrow$ (forward), $\downarrow$ (backward), $\leftarrow$ (rotated view left), and $\rightarrow$ (rotated view right). Simulated eye height was 68 virtual units $(\sim 1.73 \mathrm{~m})$. Participants selected a location by walking into it. Selection of a rewarded location resulted in auditory feedback (bell sound) and a 7-s intertrial interval (ITI) in which the monitor went black and participants progressed to the next trial. Selection of a nonrewarded location resulted in different auditory feedback (buzz sound) and required participants to continue searching.

Training Training consisted of 12 trials. Participants were randomly assigned to one of two groups: small trapezoid or large trapezoid. The gender and number of participants were balanced across groups. For both groups, the location in the top right corner was designated as the rewarded location such that searching at the egocentric right-hand side of the principal axis and at a location specified by short wall left, short wall right, and obtuse corner angle was rewarded (see Fig. 1). Participants started each trial in the center of their respective trapezoid (marked with a gray circle in Fig. 1). Participants entered their respective trapezoid-shaped enclosure at random orientations from $0^{\circ}$ to $270^{\circ}$ in increments of $90^{\circ}$.

Testing Testing consisted of 60 trials composed of 12 fivetrial blocks. Each trial block was composed of four training trials and one test trial. The order of the training and test trials was randomized within each block. For each test trial, one of four enclosures was presented: small rectangle, large rectangle, small parallelogram, large parallelogram. Each enclosure was presented once without replacement until all four had been presented. Each enclosure was presented three times (total of 12 test trials). Participants made one response during test trials, which resulted in no auditory feedback, followed by the 7-s ITI and progression to the next trial. 
Participants entered all enclosures during testing in the center of the enclosures (marked with gray circles in Fig. 1) at random orientations from $0^{\circ}$ to $270^{\circ}$ in increments of $90^{\circ}$.

\section{Results}

Training

Figure 2 (top panel) shows the mean proportion of participants' correct first responses plotted by three-trial blocks for the twelve trials of training. As shown, participants learned to respond to the rewarded location (i.e., correct location) by the end of training to an equivalent level of accuracy. A two-way mixed ANOVA on mean proportion of correct first responses with group (trained in small trapezoid, trained in large trapezoid) and block (1-4) as factors revealed a main effect of block, $F(3,102)=19.09, p<.001$, a main effect of group, $F$ $(1,34)=5.23, p<.05$, and a significant Group x Block interaction, $F(3,102)=3.63, p<.05$. The significant interaction resulted from no difference between the groups for Blocks 1, 2, and 4, independent-samples $t$ tests, $t \mathrm{~s}(34)<1.7$,
Fig. 2 Top panel: Mean proportion of participants' correct first responses plotted by three-trial blocks for the 12 trials of training for both groups. Bottom panel: Mean proportion of responses to locations specified by the trained side of the principal axis (i.e., top-right and bottom-left locations) for both groups plotted by enclosure type. Dashed lines represent chance performance. Error bars represent standard errors of the means
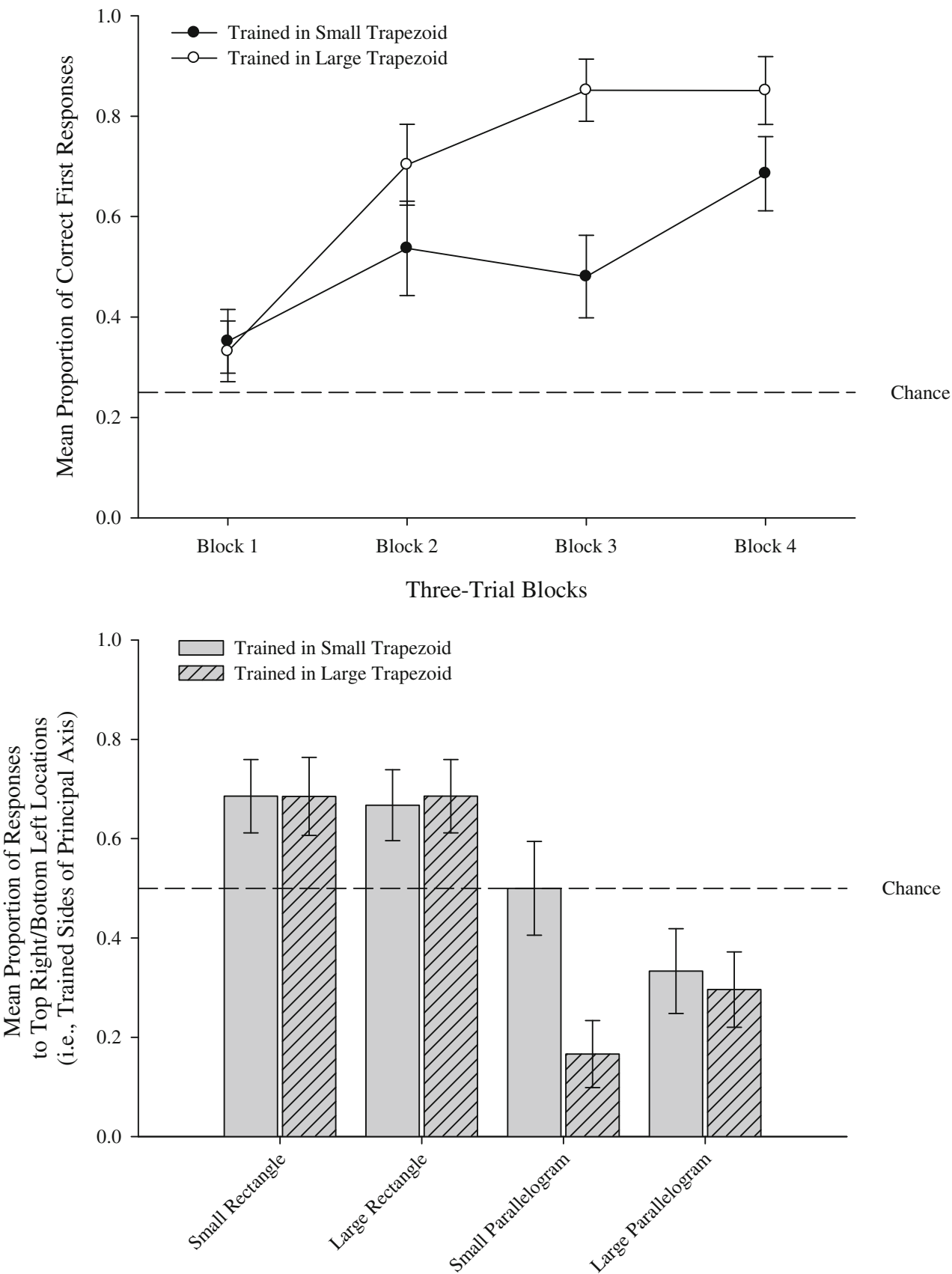

Testing Enclosure Type 
$p \mathrm{~s}>.1$, but a difference between the groups during Block 3, independent-samples $t$ test, $t(34)=3.62, p<.01$. It should be noted that Block 1 was not significantly different from chance performance (i.e., 0.25) for either group, one-sample $t$ tests, $t \mathrm{~s}$ $(17)<1.62, p s>.11$, but Blocks $2-4$ were each significantly greater than chance performance for both groups, one-sample $t$ tests, $t \mathrm{~s}(17)>2.83, p \mathrm{~s}<.05$.

\section{Testing}

Figure 2 (bottom panel) shows the mean proportion of responses to top-right and bottom-left locations (i.e., locations specified by the trained sides of the principal axis) plotted by enclosure type for both those trained in the small trapezoid and those trained in the large trapezoid. A twoway mixed ANOVA on mean proportion of responses to top-right and bottom-left locations (i.e., locations specified by the trained sides of the principal axis) with group (trained in small trapezoid, trained in large trapezoid) and enclosure type (small rectangle, large rectangle, small parallelogram, large parallelogram) as factors revealed a main effect of enclosure type, $F(3,102)=25.06, p<.001$, and a significant Group x Enclosure Type interaction, $F(3,102)=4.03, p<$ .01 . The effect of group was not significant, $F<1.1, p>.3$. The source of the interaction resulted from no difference between the groups in either the small rectangle, large rectangle, or large parallelogram, independent-samples $t$ tests, $t \mathrm{~s}(34)<0.4, p \mathrm{~s}>.75$, but a significant difference between the groups in the small parallelogram, independent-samples $t$-test, $t(34)=2.88 p<.05$.

We also compared each enclosure type to chance performance (i.e., 0.5) for both groups. Performance in the small and large rectangles was significantly above chance for both groups, one-sample $t$ tests, $t \mathrm{~s}(17)>2.35, p \mathrm{~s}<.05$. However, performance in the small and large parallelograms was not significantly different from chance for the trained in small trapezoid group, one-sample $t$ tests, $t \mathrm{~s}(17)<1.95, p \mathrm{~s}>.05$, but was significantly below chance for the trained in large trapezoid group, one-sample $t$ tests, $t \mathrm{~s}(17)>2.67, p \mathrm{~s}<.05$. It should be noted that the at-chance performance by the participants in the trained in small group reflects that of the responses of most participants as opposed to a dichotomous split (as reflected by the size of the error bars). These results suggest that in the absence of spatial cues other than the principal axis of space (i.e., in the rectangles) participants in both groups were able to utilize this spatial cue for orientation to an equivalent, above chance level. Moreover, these results suggest that neither training nor testing size influenced the use of the principal axis of space (a global spatial cue) for orientation. In contrast, when trained corner angles and the trained sides of the principal axis of space were in conflict (i.e., in the parallelograms), participants were differentially influenced by these spatial cues. Specifically, participants trained in the small trapezoid relied on trained corner angles and the trained sides of the principal axis of space to an equivalent extent, whereas participants trained in the large parallelogram relied on the trained corner angles to a greater extent than the trained sides of the principal axis of space. Collectively, these results indicate that enclosure size influenced the use of corner angles (a local spatial cue) but not the principal axis of space (a global spatial cue) for orientation.

\section{Discussion}

Regardless of training or testing enclosure size, participants allocated responses to the trained sides of the principal axis at above-chance levels in the rectangular-shaped enclosures. Importantly, participants in both groups allocated responses to these locations to an equivalent level. However, when trained corner angles and the trained sides of the principal axis were in conflict (as they were in the parallelograms), participants trained in the small trapezoid allocated responses to these locations to an extent that was not different from chance. In contrast, participants trained in the large trapezoid allocated more responses to the locations specified by the trained corner angles than to the trained side of the principal axis of space (i.e., performed significantly below chance) in the parallelograms. In short, the trained corner angles exerted more influence on the responses of participants who were trained in the larger enclosure when they were placed in conflict with the principal axis of space.

Importantly, the nature of the training and testing enclosures allowed us to rule out alternative spatial cues other than corner angle and the principal axis of space for reorientation. Specifically, the training enclosures allowed us to render wall lengths irrelevant because participants were trained to both the left and right side of a particular wall length. The rectangular testing enclosures allowed us to isolate the use of the principal axis of space because wall lengths were rendered irrelevant from training and corner angles were not only novel but also equivalent. The parallelogram testing enclosures allowed us to place trained corner angles and the trained sides of the principal axis of space in direct conflict (again because wall lengths were rendered irrelevant in training).

The present results appear consistent with those obtained when investigating the influence of enclosure size on the use of feature and geometric cues (Ratliff \& Newcombe, 2008; Sovrano et al., 2005, 2007; Sovrano \& Vallortigara, 2006; Vallortigara et al., 2005). Our results suggest that, much like feature cues, local geometric cues also appear to exert a greater influence in larger enclosures than in smaller enclosures. Importantly, our results extend this possibility by potentially delineating the source of the relative changes in 
spatial cue use during manipulations of enclosure size. Specifically, we interpret the present results to suggest that changes in local geometric cues are the source of the relative contribution of local and global geometric cues during changes of enclosure size.

We acknowledge that we are unable to definitively state whether we are directly influencing the use of local cues (i.e., size of the enclosure increased or decreased reliance on local cues) or the use of global cues (i.e., size of the enclosure increased or decreased reliance on global cues) because our design did not allow us to isolate the use of corner angles for orientation. As an aside, we are not aware of any quadrilateral shapes that would allow us to isolate the use of only the $120^{\circ}$ local geometric cue used in the present experiment. At first blush, a rhombus would appear to be an ideal possibility, but such a shape does not eliminate (or render useless) the principal axis of space to assess the sole influence of corner angles. Despite this limitation, however, the rectangular enclosures were able to isolate the use of the principal axis of space, and results from these test trials indicate that the size of the enclosure had no influence on the use of global geometric cues. Specifically, both groups performed equally well and at above-chance levels. Given this equivalent and above-chance performance for both groups in the rectangular testing enclosures, we suggest that enclosure size is influencing the use of local but not global geometric cues. In other words, we suggest that changes in local cue use are responsible for the relative contribution of local and global geometric cues during changes of enclosure size. However, it is important to note that it remains unclear whether training size or testing size or both are exerting influence on the use of local geometric cues. Specifically, it remains unknown whether a process of extracting local geometric cues (e.g., corner angles) is relatively easier in a large environment than in a small environment and/or is relatively more difficult in small environments than in large environments.

Present results have implications for understanding the role of enclosure size on the use of feature and geometric cues. If feature and geometric cues are influenced in ways similar to those of local and global geometric cues (as the present results suggest), then our obtained results suggest that enclosure size may be directly influencing the use of feature but not geometric cues during reorientation. Such a possibility has explicit implications for existing theories of geometry learning. For example, perhaps corner angles function like independent features (see Dawson, Kelly, Spetch, \& Dupuis, 2010; Miller, 2009; Miller \& Shettleworth, 2007). Although we acknowledge that this possibility is not entirely novel (i.e., Dawson et al., 2010; Miller, 2009; Miller \& Shettleworth, 2007), our results provide clarification on terminology related to what defines a feature, suggest that corner angle (and perhaps all local geometric cues) should be categorized as features, and clarify the mechanism by which enclosure size influences the relative contribution of feature and geometric cues during changes of enclosure size.

Despite the promise of incorporating corner angles into existing models of geometry learning as discussed previously, it should be noted that these existing theoretical models continue to face difficulties in accounting for the use of the principal axis of space in orientation tasks (e.g., the abovechance performance in the rectangles by participants in both groups in the present experiment). These theoretical models appear to face this difficulty because principal axis is not considered as an independent cue (see Dawson et al., 2010; Miller, 2009; Miller \& Shettleworth, 2007); however, even if principal axis were considered as an independent cue in these models, presumably this cue should be susceptible to changes in enclosure size in a manner similar to that of other cues. As a result, our results add to a growing body of literature that require some form of a global orienting cue (e.g., principal axis of space) to account for obtained results from tasks that are devoid of alternative spatial cues for orientation (e.g., Bodily et al., 2011; Sturz \& Bodily, 2011b; Sturz et al., 2011). Perhaps, most importantly, present results provide evidence that local (and perhaps feature) cues are responsible for changes in the relative use of local (and perhaps feature) and global geometric cues across changes in enclosure size.

Author note The present research was conducted following the relevant ethical guidelines for human research and was supported in part by funds from the Office of the Vice President for Research and the Jack N. Averitt College of Graduate Studies at Georgia Southern University. The authors thank three anonymous reviewers for their helpful comments on a previous version. Correspondence may be addressed to: B. R. Sturz, Department of Psychology, Georgia Southern University, P.O. Box 8041, Statesboro, GA 30460 (e-mail: bradleysturz@georgiasouthern.edu).

\section{References}

Bodily, K. D., Eastman, C. K., \& Sturz, B. R. (2011). Neither by global nor local cues alone: Evidence for a unified orientation process. Animal Cognition, 14, 665-674.

Cheng, K. (2005). Reflections on geometry and navigation. Connection Science, 17, 5-21.

Cheng, K. (2008). Whither geometry? Troubles of the geometric module. Trends in Cognitive Sciences, 12, 355-361.

Cheng, K., \& Newcombe, N. S. (2005). Is there a geometric module for spatial orientation? Squaring theory and evidence. Psychonomic Bulletin \& Review, 12, 1-23.

Dawson, M. R. W., Kelly, D. M., Spetch, M. L., \& Dupuis, B. (2010). Using perceptrons to explore the reorientation task. Cognition, 14, 207-226.

Kelly, D. M., Chiandetti, C., \& Vallortigara, G. (2011). Re-orienting in space: Do animals use global or local geometry strategies? Biology Letters, 7, 372-375.

Kelly, D. M., Durocher, S., Chiandetti, C., \& Vallortigara, G. (2011). A misunderstanding of principal and medial axes? Reply to Sturz and Bodily. Biology Letters, 7, 649-650. 
Miller, N. Y. (2009). Modeling the effects of enclosure size on geometry learning. Behavioural Processes, 80, 306-313.

Miller, N. Y., \& Shettleworth, S. J. (2007). Learning about environmental geometry: An associative model. Journal of Experimental Psychology: Animal Behavior Processes, 33, 191-212.

Ratliff, K. R., \& Newcombe, N. S. (2008). Reorienting when cues conflict: Evidence for an adaptive combination view. Psychological Science, 19, 1301-1307.

Sovrano, V. A., Bisazza, A., \& Vallortigara, G. (2005). Animals' use of landmarks and metric information to reorient: Effects of the size of the experimental space. Cognition, 97, 121-133.

Sovrano, V. A., Bisazza, A., \& Vallortigara, G. (2007). How fish do geometry in large and in small spaces. Animal Cognition, 10, 47-54.

Sovrano, V. A., \& Vallortigara, G. (2006). Dissecting the geometric module: A sense linkage for metric and landmark information in animals' spatial reorientation. Psychological Science, 17, 616-621.

Sturz, B. R., \& Bodily, K. D. (2011a). Is surface-based orientation influenced by a proportional relationship of shape parameters? Psychonomic Bulletin \& Review, 18, 848-854.

Sturz, B. R., \& Bodily, K. D. (2011b). Of global space or perceived place? Comment on Kelly et al. Biology Letters, 7, 647-648.
Sturz, B. R., Bodily, K. D., Katz, J. S., \& Kelly, D. M. (2009). Evidence against integration of spatial maps in humans: Generality across real and virtual environments. Animal Cognition, 12, 237-247.

Sturz, B. R., Brown, M. F., \& Kelly, D. M. (2009). Facilitation learning spatial relations among locations by visual cues: Implications for theoretical accounts of spatial learning. Psychonomic Bulletin \& Review, 16, 306-312.

Sturz, B. R., Gurley, T., \& Bodily, K. D. (2011). Orientation in trapezoid-shaped enclosures: Implications for theoretical accounts of geometry learning. Journal of Experimental Psychology. Animal Behavior Processes, 37, 246-253.

Sturz, B. R., \& Kelly, D. M. (2009). Encoding of relative enclosure size in a dynamic three-dimensional virtual environment by humans. Behavioural Processes, 82, 223-227.

Sturz, B. R., Kelly, D. M., \& Brown, M. F. (2010). Facilitation of learning spatial relations among locations by visual cues: Generality across spatial configurations. Animal Cognition, 13, 341-349.

Vallortigara, G., Feruglio, M., \& Sovrano, V. A. (2005). Reorientation by geometric and landmark information in environments of different sizes. Developmental Science, 8, 393-401. 